Results After danhong injection treatment, the curative effect was better in treatment group than control group $(p<0.01)$, BNP and CRP plasma levels both decreased significantly $(p<0.01)$. In treatment group, BNP and CRP plasma levels more decreased significantly $(\mathrm{p}<0.05)$.

Conclusion Danhong injection can be the effective drug used in clinic for treating unstable angina.

\section{e0622 CLINICAL EFFECT OF SHEXIANG BAOXING PILLS ON CORONARY HEART DIEASE IN PATIENTS WITH HEART FAILURE}

doi:10.1136/hrt.2010.208967.622

Ling Han, Jin-liang Chen, Yong-guo Miao, Shi-hua Li, Guo-jun Li, Zhi-ming Liu. Department of Cardiology, Handan Railway Hospital, Hebei Province, Hebei Handan, China

Objective To observe the curative effect of Shexiang Baoxing Pills on coronary heart diease in patients with heart failure (CHF).

Methods 116 patients on coronary heart disease with heart failure were divided randomly into two groups, one was regular treatment group treated with diuretic, ACE inhibitor, $\beta$-Blockers and digitoxin, and another was Shexiang Baoxing Pills treatment group treated with Shexiang Baoxing Pills on the basis of above regular treatment. All the patients were followed up for 6 months and observed the changes of the clinical symptoms, left ventricular ejection fraction (LVEF), left ventricular end diastolic diameter (Lived), 6 min walking distance and myocardial ischaemia paroxysm count of $24 \mathrm{~h}$.

Results After Shexiang Baoxing Pills, each index of the Shexiang Baoxing Pills treatment group was obviously improved and the LVEF was higher than regular treatment group $(p<0.01)$, LVEDd and myocardial ischaemia paroxysm count of $24 \mathrm{~h}$ was reductive than regular treatment group $(\mathrm{p}<0.01)$.

Conclusion Shexiang Baoxing Pills can be the effective drug used in clinic for treating chronic heart failure.

\section{e0623 OPTIMAL LEFT VENTRICULAR LEAD LOCATION AND THE LONG-TERM OUTCOMES OF CARDIAC RESYNCHRONISATION THERAPY}

doi:10.1136/hrt.2010.208967.623

\begin{abstract}
${ }^{1}$ Ying-Xue Dong, ${ }^{1}$ Yan-Zong Yang, ${ }^{2}$ Robert F Rea, ${ }^{2}$ Brian D Powell, ${ }^{2}$ Samuel J Asirvatham. 'First Affiliated Hospital of Dalian Medical University, Dalian, China; ${ }^{2}$ The Division of Cardiovascular Diseases, Mayo Clinic, Rochester, Minnesota, and the department of Cardiology
\end{abstract}

Introduction Lateral or posteriolateral coronary vein has been considered the optimal location of left ventricular (LV) lead placement for cardiac resynchronisation therapy (CRT). However, the long-term clinical outcomes of CRT based on LV lead location have not been sufficiently addressed.

Methods Seven hundred and eighteen CRT-P/CRT-D recipients from Jan 2002 to Dec 2008 were studied. At the CRT implant, the LV lead placement was prioritised as posterolateral/lateral (PL, 50\%), anterior lateral (AL, 31\%), anterior interventricular (AI, 12\%) or middle cardiac veins (MC, 7\%). NYHA class and echocardiography were assessed before and after CRT. Clinical outcomes of CRT were compared among 4 LV lead locations.

Results Patient baseline demographics, except the gender $(\mathrm{P}=0.03)$, were similar among four groups. After CRT, the improvement in NYHA class, LV ejection fraction (EF), and LV diastolic diameter were significantly improved comparably across four LV lead locations (table). The increase in LVEF by $>5 \%$ were $46 \%, 47 \%, 41 \%$ and $42 \%$ in $\mathrm{PL}, \mathrm{AL}, \mathrm{AI}$ and $\mathrm{MC}$ groups ( $\mathrm{p}=0.87$ ).

Conclusion The present study suggests that LV lead positioned in the alternative coronary veins, other than posteriolateral or lateral location, achieve comparable benefit in improvement of HF symptoms, and LV function after CRT.

\section{E0624 THE EFFECT OF CARDIAC RESYNCHRONISATION THERAPY ON NOVEL NEUROHORMONES IN HEART FAILURE}

doi:10.1136/hrt.2010.208967.624

${ }^{1}$ Ying-Xue Dong, ${ }^{2}$ John C Burnett Jr, ${ }^{2}$ Horng H Chen, ${ }^{2}$ Sharon Sandberg, ${ }^{3}$ Yanhua Zhang, ${ }^{3}$ Peng-Sheng Chen, ${ }^{1}$ Yong-Mei Cha. ${ }^{1}$ First Affiliated Hospital of Dalian Medical University, Dalian, China; ${ }^{2}$ The Department of Cardiology, The Division of Cardiovascular Diseases, Mayo Clinic, Rochester, Minnesota, USA

Background Neurohormonal dysregulation contributes to heart failure (HF) progression. We sought to determine the effect of cardiac resynchronisation therapy (CRT) on the nerve growth factor (NGF), a biomarker that promotes the maturation, and survival of sympathetic nerve endings, and amino-terminal propeptide of type III procollagen (PIIINP), a marker of type III collagen synthesis.

Methods This prospective study enrolled 20 healthy age-matched controls and 45 consecutive patients (pts) who received CRT-D. NYHA class, distance of 6-min walk and echocardiography and plasma concentrations of NGF, PIIINP, b-type natriuretic peptide (BNP), norepinephrine (NE), epinephrine (EPI) and dopamine (DA) were measured before and 6 month after CRT. Response to CRT was defined as $\geq 15 \%$ reduction in left ventricular end-systolic volume index (LVESVI) at 6-month follow-up.

Results The baseline BNP $(557 \pm 692$ vs $47 \pm 35, \mathrm{p}<0.01)$ and PIIINP (8.22 \pm 3.76 vs $5.36 \pm 1.47, \mathrm{p}<0.01)$ were elevated in HF compared to controls, while NGF, NE, EPI and DA levels were not different. Twenty two of 45 pts (49\%) responded to CRT. The responder group demonstrated significant decrease only in BNP level $(p=0.04)$ at 6-month follow-up, parallelling with the clinical improvements (table 1). The baseline PIIINP was lower in CRT responders than non-responders $(p=0.04)$, and it correlated with the reduction of

Table Comparison of clinic outcomes before and after CRT in different LV lead locations

\begin{tabular}{|c|c|c|c|c|c|c|c|c|}
\hline & \multicolumn{2}{|c|}{ Lateral vein $(\mathbf{N}=359)$} & \multicolumn{2}{|c|}{ Anterolateral vein $(\mathrm{N}=\mathbf{2 2 6})$} & \multicolumn{2}{|c|}{ middle cardiac vein $(N=49)$} & \multicolumn{2}{|c|}{ anterior interventricular vein $(N=84)$} \\
\hline & Pre-CRT & Post-CRT & Pre-CRT & Post-CRT & Pre-CRT & Post-CRT & Pre-CRT & Post-CRT \\
\hline NYHA & $3.02 \pm 0.46$ & $2.34 \pm 0.81^{*}$ & $2.92 \pm 0.53$ & $2.34 \pm 0.89^{*}$ & $3.10 \pm 0.45$ & $2.70 \pm 0.85^{*}$ & $2.98 \pm 0.52$ & $2.43 \pm 0.86^{*}$ \\
\hline LVEF (\%) & $23.57 \pm 7.28$ & $30.82 \pm 11.67^{*}$ & $24.19 \pm 7.96$ & $30.44 \pm 12.06^{*}$ & $24.97 \pm 7.62$ & $30.72 \pm 11.43^{*}$ & $22.45 \pm 7.25$ & $28.40 \pm 11.73^{*}$ \\
\hline LVEDD (mm) & $65.71 \pm 9.12$ & $63.00 \pm 9.73^{*}$ & $65.79 \pm 8.87$ & $63.88 \pm 9.88^{*}$ & $65.39 \pm 9.32$ & $62.61 \pm 10.25^{*}$ & $68.48 \pm 8.92$ & $64.86 \pm 9.67^{*}$ \\
\hline LVESD (mm) & $57.91 \pm 9.89$ & $52.83 \pm 11.68^{*}$ & $56.69 \pm 10.23$ & $53.03 \pm 12.01 *$ & $56.18 \pm 11.56$ & $52.24 \pm 13.11^{*}$ & $59.09 \pm 10.04$ & $55.32 \pm 13.30$ \\
\hline $\mathrm{RVD}(\mathrm{mm})$ & $0.90 \pm 0.86$ & $0.82 \pm 0.85$ & $0.85 \pm 0.92$ & $0.82 \pm 0.85$ & $0.86 \pm 0.83$ & $1.01 \pm 0.84$ & $0.56 \pm 0.77$ & $0.78 \pm 0.91$ \\
\hline RV dysfunction & $1.05 \pm 0.92$ & $0.88 \pm 0.92^{*}$ & $1.03 \pm 0.93$ & $0.93 \pm 0.91$ & $1.06 \pm 0.97$ & $1.06 \pm 0.90$ & $0.74 \pm 0.86$ & $0.79 \pm 0.96$ \\
\hline MR (m/s) & $1.54 \pm 0.78$ & $1.30 \pm 0.75^{*}$ & $1.57 \pm 0.83$ & $1.35 \pm 0.75^{*}$ & $1.53 \pm 0.71$ & $1.15 \pm 0.70^{*}$ & $1.36 \pm 0.69$ & $1.19 \pm 0.68$ \\
\hline
\end{tabular}

${ }^{*} P<0.05$ compared to pre-CRT. 